\title{
UTILIZING NEURAL NETWORKS FOR CONDITION ASEESSMENT OF SANITARY SEWER INFRASTRUCTURE
}

\author{
Dulcy M. Abraham ${ }^{1}$, Myung Jin Chae ${ }^{2}$ and Sanjiv Gokhale ${ }^{3}$
}

\author{
${ }^{1}$ Associate Professor, School of Civil Engineering, Purdue University, \\ West Lafayette, IN 47907, U.S.A. \\ ${ }^{2}$ Ph.D. Candidate and Graduate Research Assistant, School of Civil Engineering, \\ Purdue University, West Lafayette, IN 47907, U.S.A. \\ ${ }^{3}$ Associate Professor, Purdue School of Engineering and Technology, \\ Indiana University Purdue University at Indianapolis, Indianapolis, IN 46202, U.S.A.
}

\begin{abstract}
This paper will describe the development of an improved methodology for accurately analyzing and interpreting data regarding the condition of sanitary sewer systems. The proposed methodology enables fast and accurate assessment, which is significant in building a sewer condition database for asset management. The inspection system obtains optical data from the Sewer Scanner and Evaluation Technology (SSET). Multiple neural networks are developed to classify the pipe defect features and a fuzzy logic system suggested to filter and fusion the multiple neural network outputs.
\end{abstract}

Keywords: Sewer system, SSET, condition assessment, inspection, artificial neural networks, defect detection, image processing, pattern recognition.

\section{INTRODUCTION}

Detection of interior defects in sewer pipelines is the first warning of problems that could occur within sewer lines. Internal inspections are commonly performed through three methods, i.e., physical inspection, photographic inspection, and CCTV inspection. Physical inspection involves direct man-entry inspection of larger sewers not in service. Photographic inspection utilizes a camera to take a series of color photographs along the inside of sewer lines. At present, the state-of-the-practice inspection systems are dominated by closed-circuit TV (CCTV) technology, in which remotely controlled vehicles carry a TV camera through the sewer pipes. Diagnosis of failures or defects depends on the experience, the capability and the concentration of the operator, and the reliability of the TV picture. Detection of subtle defects and assessment of the degree of deterioration are particularly error prone, so the method is adequate only for detecting gross defects.

\section{ADVANCEMENTS IN CONDITION ASSESSMENT OF SEWER SYSTEMS}

A large number of automated data collection technologies such as photographic and video imaging, laser, radar and infrared thermography have made it possible to collect data in shorter time for the analysis of infrastructure performance. However, the accuracies and precisions of these technologies are not well understood, and infrastructure managers and planners have been reluctant to adopt them in practice.

Some promising non-destructive, diagnostic methods have recently been developed for condition assessment of sewer systems. In Japan, the Sewer Scanner and Evaluation Technology (SSET) was developed jointly by TOA Grout, CORE Corporation and TGS Company. This break-through technology in sewer condition assessment is a flexible data acquisition tool for non-destructive evaluation. More and higher quality information regarding sewer conditions can be obtained by utilizing SSET's combination of Closed Circuit Television (CCTV) technology, an optical scanner, and gyroscopic technology. The SSET provides information including a CCTV video record, a full circumference scanned image of the pipe, a computer generated color coded print-out of the defects, a written description of each defect along the pipe (based on inspector reports), and horizontal and vertical pipe deflections [1], [5], [11]. 


\section{AUTOMATED INTERPRETATION FOR SEWER ASSESSMENT}

Currently, the reliability of the condition assessment is low due to the subjective interpretation of images. The task to be performed by interpretation system is complex because there are many types of defects (e.g., leaking joints, cracks, root intrusions, corrosion, etc.) In addition, some of these defects may also overlap.

One of the standard techniques used in image processing is the two-dimensional Fourier transform which converts an image into its frequency components. Many of the important features in the original image can be identified and classified from the amplitude and phase of the frequency components. But the use of Fourier transforms for defect detection is not very successful for defect detection when: (a) the relation between a defect and frequency components are not known, (b) the quality of the images (e.g., signal-to-noise ratio) used for detection may be low, and (c) the problem is very complex. In such cases, neural-network based pattern recognition algorithms are preferable [3].

Artificial Neural Networks (ANN) are associated with storage, knowledge base, and the recognition that correspond to high-level processing. Neural networks are able to create new information processing functions, such as specific feature detectors and ordered internal representations for structured signals, in response to frequently occurring signal patterns. Also, neural networks can create higher abstractions (symbolisms) from raw data completely automatically. Intelligence in neural networks ensues from abstractions, not from heuristic rules or manual logic programming [6].

The procedure for image analysis and recognition, that was used for sanitary sewer assessment using neural networks, is divided into four steps, namely, image acquisition, preprocessing, defect recognition using multiple neural networks, and fuzzy estimation, as shown in Figure 1.

\subsection{Image Acquisition}

While the SSET travels through the pipelines with the speed of 3 to 4.5 meters per minute, it transfers the scanned image which is continuous from the starting point to the ending point along with gyroscope data. A prominent advantage of this system is that this system does not have to stop roving for the inspector to mark and take a close look on every encountered defect. This reduces the time of fieldwork greatly.

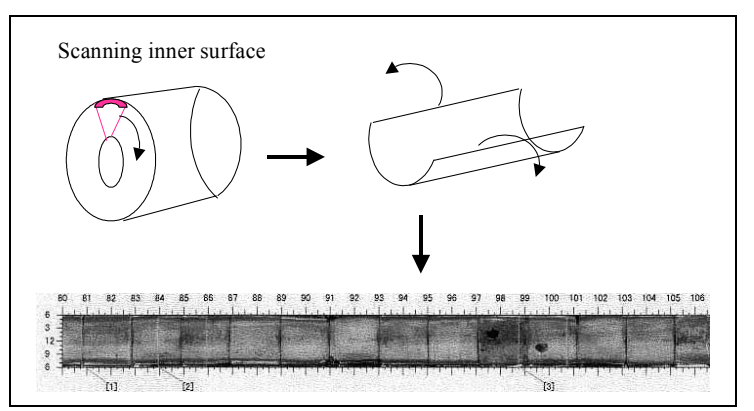

Figure 2. Image transferred by SSET

The circumference scanned image is unwrapped and stored in a digital form in floppy disk of CD-ROM (Figure 2). Once the inner surface image and gyroscope data are stored in floppy disk or CD-ROM format, the disk is delivered to the engineering office for the interpretation of the data and assessment of pipe condition. Since this inspection method does not require on-site interpretation, the amount of field work is greatly reduced. 
Another advantage of collecting data using the SSET is that the distance from the sensor to the object (pipe wall) is the same all across the scanned area Moselhi et. al. [8] utilized ordinary CCTV images for crack detection. In that case, the CCTV images had to be converted to account for the varying distance from the camera to the objects. However, converting 2D scanned images onto a 3D coordinate system is challenging. Since images delivered by the SSET are taken from the same distance from the sensor, the third coordinate (distance from the camera to the objects) does not have to be considered.

\subsection{Preprocessing}

The main purpose of preprocessing is to modify the original image into "smaller" elements that can be handled with greater ease. Preprocessing of image the size of the input data is very large - (24-bit) times $638 \times 1000$ (the size of the input image). This requires a very large number of input nodes in the ANN, which translates to high computing times. Thus, in order to reduce computing times, some modification of the original images is needed.

The original $638 \times 1000$ image size required 638,000 neurons in the input layer. The original image was reduced by one quarter of the original image size to increase the computing speed and to avoid the restrictions of system memory. The images were digitized into two dimensional matrices (159 x 250), in which each row and column identifies a point in the image and the corresponding matrix element value represents the gray level at that point. Each pixel in such a digitized array has a 24-bit color depth, which can store up to 16777215 different colors.

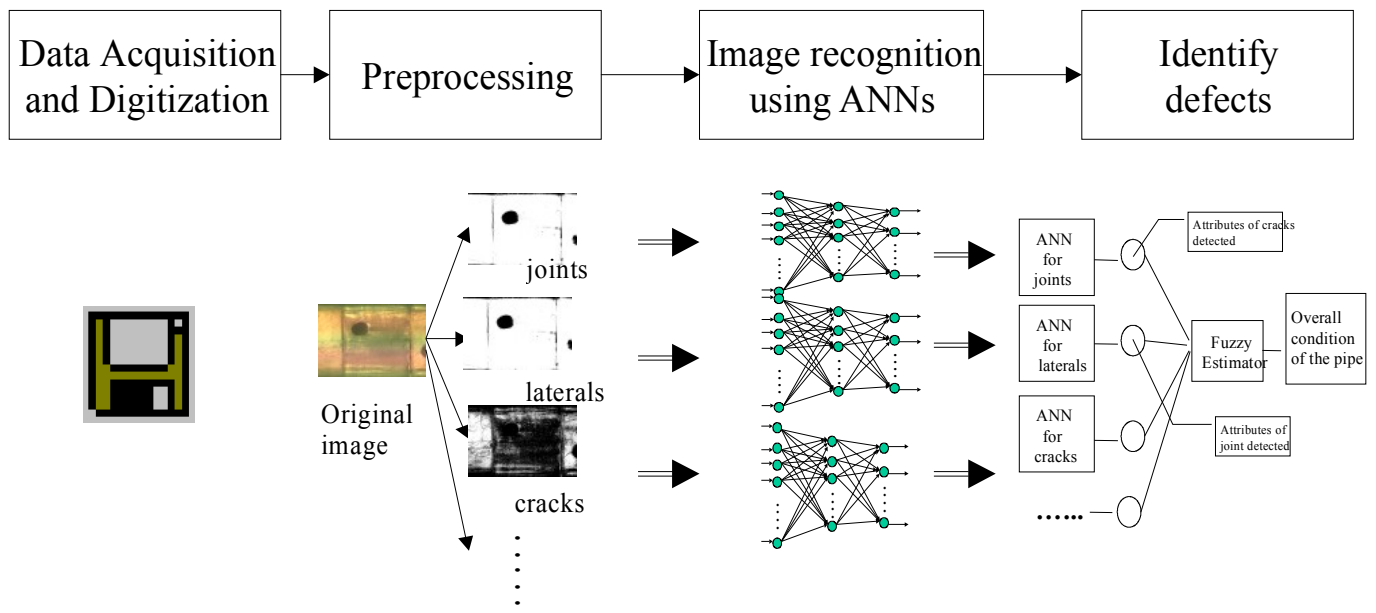

The original color image is changed into gray-level image and binarized through binary transformation in order to discard background noise. Then the transformed image is converted into an edge detected image using the edge detection algorithm provided by Matlab Version 5.

Figure 1. Image ana lysis and recognition using ANN means early vision processing or low-level processing [7]. Appropriate preprocessing is very important in order to make images suitable for various purposes. For example, an image can be preprocessed to make it distinguishable to human eye and/or more appropriate for computing purpose. The processing does not create new information from the original information. It sharpens the image feature, adjusts contrast, etc [2].

Through filtering, gray scale manipulation, isolation of regions, restoration, etc., the acquired images are treated in order to make them amenable for treatment by artificial neural networks. The original image is divided into several sub-images and treated so that each sub-image shows one type of defects (joints, cracks, corrosion, etc), as shown in Figure 3. The input image data is converted into binary format so that it is easier to "program" the network, and faster computer processing speed is expected.

Theoretically, original images could be used by the ANN without preprocessing. In this case, however,

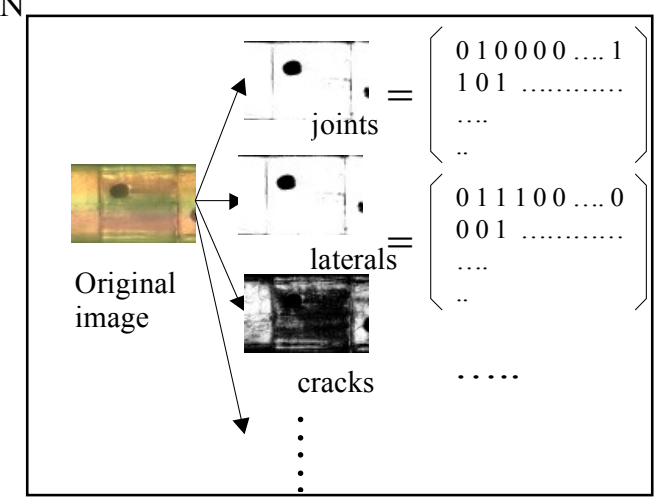

Figure 3. Isolation of defects

\subsection{Defects recognition using multiple neural networks.}

Backpropagation neural networks are recognized for their superior performance in pattern recognition [4], [8], [9]. Since the sets of target data and data related to sewer pipe (in the form of SSET images) are available, the supervised learning method is 
appropriate for the sewer condition assessment problem. In this case, supervised learning method with backpropagation algorithm is used.

After the images are preprocessed, neural networks are used to identify and recognize the type and level of the defects in the pipeline (Figure 4). A network is "created" for each type of defect. For example, ANN 1 is developed for detecting joints and ANN 2 for detecting corrosion. Outputs of a network are the attributes of a defect. For example, the set of outputs of an ANN for joint recognition "displays" attributes of joints such as number of joints, width of joints, etc (as shown in Figure 4). In the same manner, the attributes of cracks may be defined as the number of cracks in the projected section, nature of cracks, etc.

In supervised learning method, target values should be set for the training of an ANN. Target values are known attributes of a defect. The ANN is trained with the known target values. This means that the outputs of a network, which are attributes of a defect, are compared with the target values and a network is trained by adjusting the weight factors in the network. Once the networks are trained, they are tested and verified with known defects and they are ready to be used for the new scanned data for the assessment.

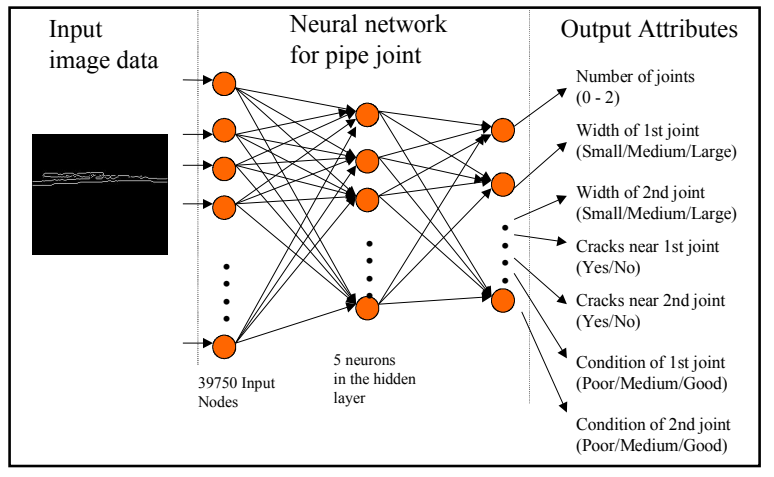

Figure 4. Example: ANN for joint detection

In the preprocessing stage, to facilitate the classification of defects from the background image, feature extraction techniques are employed. For example, while Figure 5 (a) shows the original image obtained from SSET, Figure 5 (b) and Figure 5 (c) show an edge detected image and the corresponding extracted features, respectively. The image 5 (a) is first changed to gray scale image and then used in the edge detection algorithm. Detected edges are represented by numbered features (Figure 6). The characteristics of the numbered features are also analyzed. Approximately 63 characteristics including size, perimeter, breadth, elongation, etc., can be identified.

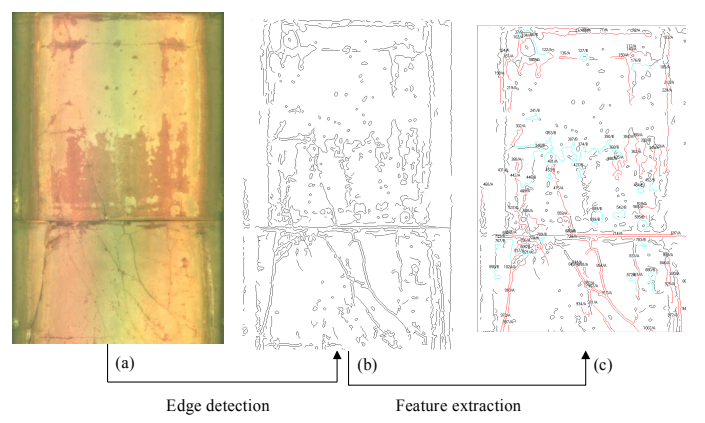

Figure 5. Preparation for ANN training

By inspecting the characteristics of detected features, they can be categorized into crack, joint, service lateral, or noise. As there are many characteristics to inspect, neural networks are employed to find patterns in the characteristics of edge-detected features.

\subsection{Neural network-driven fuzzy system}

It is beneficial to use fuzzy membership functions and fuzzy logic to describe real world information that is untamed or "noisy." [12]. The outputs obtained from neural networks have those characteristics. The outputs of neural networks are generally huge series of meaningless numbers that are difficult to interpret. By combining neural network and fuzzy logic, outputs of neural networks are filtered to produce smooth outputs. Fuzzy systems filter undesired information, and thereby improve signal-to-noise ratio [10]. Thus, for more realistic and accurate assessment results, neuralnetwork-driven fuzzy logic system is suggested. Adding fuzzy rule-base system to neural network outputs makes this filtering process feasible by assuming that the neural network's outputs are fuzzy membership values for fuzzy system (Figure 7).

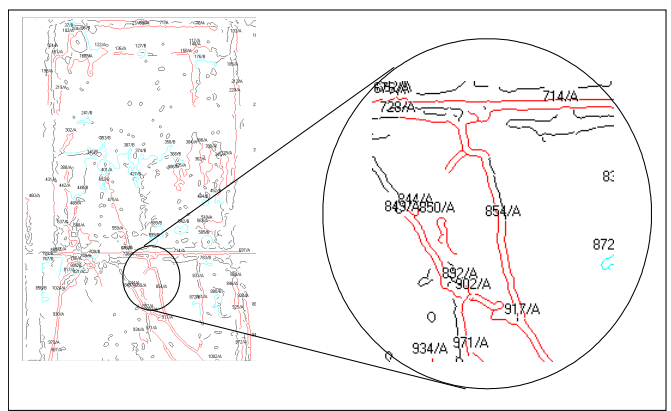

Figure 6. Detailed picture

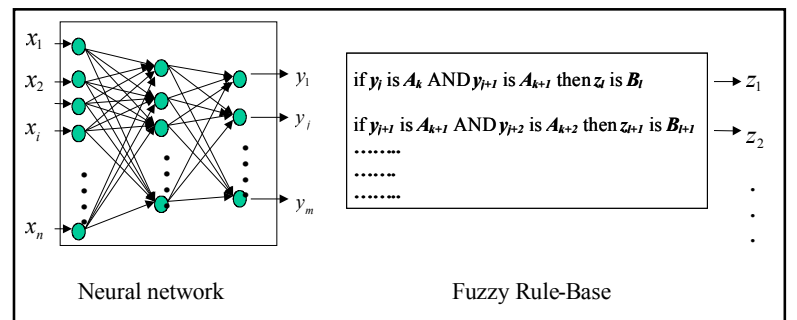


Figure 7. Schematic of neural-network-driven fuzzy logic system

\section{TEST OF PROTOTYPE MODEL}

The proposed assessment methodology was validated using SSET data from sanitary sewer infrastructure in the city of San Jose, California. Preprocessing was done to obtain the edge detected binary image which became the input to the trained neural network. Attributes were assumed in order to describe the condition of pipe joint appropriately.

Feedforward neural network with backpropagation algorithm was used in the validation. Twenty image files were utilized to train the network. While training the network, target values suggested by only one expert are used. This led to more consistent outputs for defect recognition, ensuring consistency in the condition assessment of the pipes.

The neural network detected the number of pipe joints with $100 \%$ accuracy and the conditions of joints were predicted with $82 \%$ accuracy. This pilot model achieved $73 \%$ accuracy in detecting the existence of cracks near joints. This sufficiently proves that neural networks are capable of detecting cracks as well as joints.

\section{CONLUSIONS}

In this paper, an automated data interpretation methodology for assessing the condition of sanitary sewers was described. The combination of neural network and fuzzy logic system used for developing this infrastructure assessment tool made it possible to solve the complex pattern recognition problems. Once fully developed, the data interpretation methodology will assist asset managers in obtaining accurate and reliable information regarding current condition of sewer systems within limited time and budget.

\section{ACKNOWLEDGMENTS}

This research was primarily funded by a grant from the Purdue Research Foundation and in part by grant CMS-9973509 from the National Science Foundation. Their support is gratefully acknowledged. Any opinions, findings, conclusions, or recommendations expressed in this study are those of the writers and do not necessarily reflect the views of the National Science Foundation nor of the Purdue Research Foundation. The writers would thank the Dr. Tom Iseley, Iseley Enterprises and Dr. Kenzi Karsaki, CORE Corporation for providing the SSET data used in the validation.

\section{REFERENCES}

[1] Abraham, D. M. , Iseley, T., Prasanth, R. K. and Wirahadikusumah, R., "Integrating Sensing Technologies for Underground Utility Assessment," ASCE Conference on Infrastructure Condition Assessment: Art, Science, Practice, Boston, Massachusetts, August, pp. 316-325, 1997.

[2] Bow, S. (1992) Pattern Recognition and Image Preprocessing, Marcel Dekker, Inc. New York

[3] Hecht-Nielsen, R. (1990). Neurocomputing, Addison-Wesley, Reading.

[4] Hegazy, T., Fazio, P. Moselhi, O. "Developing Practical Neural Network Applications Using BackPropagation," Microcomputing in Civil Engineering, Vol. 9, No. 2, ASCE, Elsevier, N.Y., pp. 145-159, 1994

[5] Iseley, T., Abraham, D. M. and Gokhale, S., "Condition Assessment of Sewer Systems," Proceedings of the ASCE Conference on Trenchless Pipeline Projects: Practical Applications, Boston, Massachusetts, June 8-11, pp. 43-51, 1997.

[6] Kohonen, T., Self-Organization and Associative Memory, Springer-Verlag, New York, 1997.

[7] Kulkarni, A. D. Artificial Neural Networks for Image Understanding. Van Nostrand Reinhold, New York, NY., 1994.

[8] Moselhi O. and Shehab-Eldeen T., "An AI-Based System for Detection and Classification of Defects in Sewers," INFRA '99 International, Nov. 1999, Montreal, Canada, Center for Expertise and Research on Infrastructures in Urban Areas (CERIU), Section 3B, 1999.

[9] Takagi, H., Susuki, N., Koda, T., and Kojima, Y. "Neural Networks Designed on Approxiamte Reasoning Architecture and Their Applications," IEEE Transactions on Neural Networks, Vol 3, No. 5, pp. 752-760, 1992.

[10] Tsoukalas, L. H. and Uhrig, R. E., Fuzzy and Neural Approaches in Engineering, John Wiley \& Sons, Inc., New York, 1997.

[11] Wirahadikusumah, R., Abraham, D. M., Iseley, T., and Prasanth, R. "Assessment Technologies for Sewer System Rehabilitation," Journal of Automation in Construction, Vol. 7, No. 4, Elsevier Science Publishers, pp. 259-270, 1997.

[12] Zadeh, L. A. Theory of Fuzzy Sets, Electronics Research Laboratory, College of Engineering, University of California, Berkeley, CA., 1997. 
002_TA3.doc- 6 - 